\begin{abstract}
Mathematics anxiety is known to be detrimental to mathematics learning. This study explored if an embodied agent could be used to help alleviate student anxiety in classrooms. To examine this potential, agent-guided algebra lessons were developed, in which an animated agent was equipped with prescriptive instructional guidance and anxiety treating messages. The lessons were deployed in regular mathematics classrooms, one lesson per day over a week, with 138 boys and girls in the $9^{\text {th }}$ grade in the United States. After taking the weeklong agent-based lessons, students decreased in their mathematics anxiety $(p=.042)$ and increased in mathematics learning $(p=.001)$, regardless of the presence or absence of the agents' anxiety messages. The presence of the agents' messages only seemed to make a difference for high-anxiety students. This finding suggests that an embodied agent could provide affective support for students with special needs.
\end{abstract}

Keywords: embodied agents, mathematics anxiety, advanced learning technologies, interactive learning environments, and pedagogical agents. 


\section{An Embodied Agent Helps Anxious Students in Mathematics Learning}

Over the last three decades, some researchers in mathematics education have deliberated about affective factors that might interfere with students' learning of mathematics. Mathematics anxiety is one such factor that has been well examined. Mathematics anxiety has a detrimental effect on mathematics-related affect and performance (Hembree, 1990; Morsanyi, Busdraghi, \& Primi, 2014). Studies have also revealed mixed results on a gender difference in mathematics anxiety (Ashcraft, 2002; Devine, Fawcett, \& Szucs, 2012). Some researchers argue that girls in general have a higher level of mathematics anxiety than boys (Osborne, 2001), but others argue for no actual difference between boys and girls (Goetz, Bieg, Lüdtke, Pekrun, \& Hall, 2013).

Understanding anxiety and its effects on mathematics students is important because there is a positive relationship between students' sense emotional security and students' success in school across grade levels.(Reyes, Brackett, Rivers, White, \& Salovey, 2012). Nonetheless, there has been very little research on anxiety management in a classroom setting, and as a result, many students who have high mathematics anxiety seem to receive little help in classrooms. Due to this lack of school support, some students from affluent families might resort primarily to oneon-one clinics but those without means to hire a private tutor may be left to simply struggle on their own (Dugas \& Robichaud, 2006; Foss \& Hadfield, 1993; Hembree, 1990; Wadlington, Austin, \& Bitner, 1992). It is important for all students--regardless of socioeconomic status, gender, or race--to have the best possible chance for success, and this study has explored the use of an advanced technology, an embodied agent (an animated, on-screen character acting as a tutor), as a supplemental tool in supporting students both cognitively and emotionally. An agent can be designed to simulate an effective in-person clinical approach and help anxious students to cope with anxiety while learning mathematical concepts. In this paper, we present the 
development of an agent-based anxiety treatment and the empirical evidence for its impact that was collected through a classroom-based experiment with $9^{\text {th }}$ grade boys and girls.

\section{Theoretical Background}

\section{Mathematics Anxiety}

Mathematics anxiety is generally defined as unpleasant feelings of tension or fear when doing mathematics (Ashcraft, 2002; Ma \& Xu, 2004). The classic anxiety study identifies two components of mathematics anxiety: emotional and cognitive (Liebert \& Morris, 1967). The emotional component explains nervousness, tension, fear, and discomfort, which are manifested by physiological signals and feelings (Adams, 2001; Morris, Davis, \& Hutchings, 1981). The cognitive component involves an individual's concerns about their performance, self-doubt, and lack of confidence, and this cognitive aspect of anxiety is known to interfere with mathematics performance more than the emotional component (Morris et al., 1981). On the whole, there is a negative relationship between mathematics anxiety and mathematics achievement and mathematics-related activities (Leppavirta, 2011; Ma \& Xu, 2004). Students with high mathematics anxiety typically receive lower scores in mathematics, compared to those with low mathematics anxiety (Cates \& Rhymer, 2003; Hembree, 1990; Ma, 1999). Highly anxious students seem to build a negative view of doing mathematics and avoid taking mathematicsrelated courses (Goetz et al., 2013).

After an examination of national data from a senior cohort, Osborne (2001) reports that gender difference in mathematics anxiety partly explains gender difference in mathematics achievement. In recent studies, more girls than boys report that they experience anxiety and discomfort while learning mathematics (Devine et al., 2012; Frenzel, Pekrun, \& Goetz, 2007; Geist, 2010). Even when some girls' performances are equal to or better than boys during early 
school years, the girls show less positive affect in mathematics (e.g., low interest and weak confidence) than boys as they advance to upper grades (Baker, 2002; Brownlow, Jacobi, \& Rogers, 2000; Lindberg, Hyde, Petersen, \& Linn, 2010). In contrast, in a study conducted with German students, Göetz, et al. (2013) claim that although girls report more mathematics anxiety on generalized assessments, they do not actually experience more anxiety than do boys in mathematics classes and examinations. Göetz, et al. argue that general questionnaires might reflect girls' inaccurate beliefs about their mathematics ability, leading to their inaccurate evaluations of their anxiety.

Although the cause of mathematics anxiety is still unclear, some researchers believe that poor teaching is one source of the anxiety (Preis \& Biggs, 2001) and argue that the instructor's willingness to help anxious students is crucial for reducing mathematics anxiety in classrooms (Steele \& Arth, 1998; Wadlington et al., 1992). It is likely that anxious learners would benefit from a patient, understanding instructor, but they might not have access to such support; even the best teachers can struggle to meet the needs of every student in every class period. To fill this gap, the authors explored the potential of an embodied agent tutor as a supplemental tool.

\section{Embodied Agents in Computer-Based Learning}

Embodied agents are animated, digital characters embedded in computer-based learning to assist learners. Depending on its functionality and behaviors, a character may also be called a pedagogical agent (Johnson, Rickel, \& Lester, 2000), a virtual peer (Ryokai, Vaucelle, \& Cassell, 2003), or an embodied conversational agent (Nass, Roles, \& Wang, 2005). Over the last two decades, researchers from various disciplines have examined the impact of embodied agents on learners' affect and learning gains (Atkinson, 2002; Baylor \& Kim, 2005; Dunsworth \& Atkinson, 2007; Kim \& Wei, 2011; Moreno, Mayer, Spires, \& Lester, 2001; Wang et al., 2008). 
Overall, there is a consensus that agent presence enables computer-based learning to be more social and natural and, consequently, could promote learners' positive affect and motivation toward the learning task (Gulz, 2004; Heidig \& Clarebout, 2011; Krämer \& Bente, 2010). For instance, after working with an agent, adolescent students evaluated their learning experience as more enjoyable and more engaging (Lester et al., 2001; Mulken, Andre, \& Muller, 1998) and perceived the learning environment as easy to use (Moundridou \& Virvou, 2002). Additionally, when they worked with an agent, elementary-school children expressed a desire to use the environment again (Robertson, Cross, Macleod, \& Wiemer-Hastings, 2004). Middle-school students also demonstrated higher interest in the material and higher motivation to continue with the learning task (Moreno \& Mayer, 2000) when they worked with an agent.

More recently, researchers in agent technology have further attempted fostering an agent's affective roles for increasing students' positive emotions while learning (Graesser et al., 2008; Kim \& Lim, 2013; Krämer, Kopp, Becker-Asano, \& Sommer, 2013). We expected that a human-like agent might be able to help anxious learners to overcome their mathematics learning anxiety. It was not clear prior to this study whether an agent's carefully-designed instructional guidance would be sufficient to assist anxious students, given the social and motivational nature of an embodied agent (Baylor, 2011), or whether the addition of the agent's messages to reduce anxiety reducing would be necessary.

Purpose of the Study

The purpose of the study was to examine the effectiveness of an agent's anxiety reducing messages on learners' mathematics anxiety and their mathematics learning. In the agent-guided algebra lessons that were implemented in natural classrooms, a tutor agent was designed to help 
students to manage their anxiety through both instructional guidance and anxiety reducing messages.

\section{Research Questions}

Three sets of research questions on mathematics anxiety and learning guided the study. The first set of questions asked about the effects of an agent's anxiety messages and learner gender: 1-1) Do the anxiety messages and learner gender affect learners' mathematics anxiety? 1-2) Do the anxiety messages and learner gender affect learners' mathematics learning? Learner gender was included as a factor because gender difference has been a popular issue in mathematics anxiety research. The second set of questions asked about the effects of the agent's messages and learners' prior anxiety levels: 2-1) Do the anxiety messages and learners' prior anxiety levels affect their mathematics anxiety? 2-2) Do the anxiety messages and learners' prior anxiety levels affect their mathematics learning? Prior mathematics anxiety was included as a factor with the consideration that the effectiveness of anxiety treatment might differ among the learners who have different degrees of mathematics anxiety. The third set of questions asked about the effects of the agent's messages and learner gender for high-anxiety students: 3-1) Do the anxiety messages and learner gender affect highly anxious learners? 3-2) Do the anxiety messages and learner gender affect highly anxious learners' mathematics learning?

\section{Method}

\section{Participants}

Participants were $9^{\text {th }}$ grade boys and girls enrolled in Algebra I in a high school located in a mountain-west state of the USA. One hundred sixty-one students started the first lesson, among which twenty-three students missed at least one lesson because of tardiness or absence. A total of one hundred and thirty-eight students were included in the data analysis. In the participating 
school district, students were allowed to take Algebra I from the $7^{\text {th }}$ grade and required to complete the course by the $9^{\text {th }}$ grade. The study was implemented as regular class activities using the portable lab in the school (34 laptop computers designated to mathematics classes). The average age of the students was $15.91(S D=0.95)$. Sixty-four students $(46.4 \%)$ were male, and seventy-four students (53.6\%) were female. Prior to participation, parental consent was obtained.

\section{Material: Agent-Based Anxiety Treatment}

\section{Curricular content}

The agent-based lessons consisted of four online self-instructional modules, each module taking one class hour. The curriculum covered four topics in fundamentals of algebra: signed number arithmetic, combining like terms and distribution, factoring, and graphing linear equations using slope and y-intercept. Each lesson consisted of 4 to 5 sections dealing with component concepts, and each section consisted of two learning phases of Review and Problem Practice. In Review, the agent presented a brief review of the concepts and examples, which the students had already learned from their teacher. In Problem Practice, the students practiced solving problems guided by the agent who provided informational feedback on their performance. Figure 1 presents screen excerpts of the agent-based lessons. During the problem solving practice, the students were encouraged to use scratch paper and pencils to reach the solution and choose their answers from one out of four possible choices. The lessons were prescriptive, and the agent's feedback was responsive according to predefined error types.

\section{Agent instructional guidance}

All participating students received an agent's instructional guidance. The agent Chris was designed to be a female teacher capable of explaining the concepts proficiently. The female gender was chosen based on literature that a female agent was more popular among both male 
and female $9^{\text {th }}$ graders (Kim, 2009; Kim \& Wei, 2011). A human teacher voice was recorded for Chris. Figure 2 overviews the design of interactions between Chris and the learner. Chris used two distinct instructional strategies to help anxious students, well-organized instruction and immediate, corrective feedback. Well-organized, guided instruction is likely to assist learners with understanding mathematical concepts more effectively than less organized, discoveryoriented approaches (Haak, HilleRisLambers, Pitre, \& Freeman, 2011; Kirschner, Sweller, \& Clark, 2006; Norwood, 1994; Preis \& Biggs, 2001). In the current study, Chris presented the concepts from simple to complex and the problems from easy to difficult in a hierarchical order. Also, previous research indicated that corrective feedback had a significant positive impact on reducing mathematics anxiety (Aksu \& Saygi, 1988) and that assisting students to conceptualize why an answer was correct (more than simply providing the correct answer) helped them develop a deeper understanding and promoted their motivation to learn (Corbalan, Kester, \& van Merriëboer, 2009; Hattie \& Timperley, 2007; McKendree, 1990). Immediate feedback during problem practice was more effective for retention of information than delayed feedback (Dihoff, Brosvic, Epstein, \& Cook, 2004). So Chris provided immediate, corrective feedback as the students progressed. The algebra teachers in the participating school helped identify the common error types observed in classrooms and write the error-specific feedback messages later implemented by Chris. Agent messages to reduce anxiety

The agent's anxiety messages were designed to encourage the student to be aware of their mathematics anxiety, cope with uncertainty, and face challenges (Dugas \& Robichaud, 2006; Foss \& Hadfield, 1993). Chris adopted a well-known cognitive-behavioral approach developed by Dugas and Robichaud (2006), which included, 1) helping the person be aware of his/her 
anxiety, 2) developing a greater tolerance for uncertainty, and 3) encouraging him/her to cope with problematic situations rather than avoid them. Earlier, Foss and Hadfield (1993) recommended a similar approach to treat mathematics anxiety in one-on-one settings: 1) helping the student to identify their fears and the source of the fears, and 2) suggesting coping skills to build confidence. Each time a student was about to progress to the next section in a lesson, an anxiety question was presented, and the student had to answer the question in order to move on. Four to five anxiety questions were asked depending on the number of subsections in the lesson: one question in a multiple-choice format with four choices and the rest in a Yes/No format. The agent's responses to the choices were pre-scripted, and given the limited choices that students had, the authors did not view the agent's responses as adaptive. For example, Chris asked a learner, "When you solve mathematics problems, what do you usually do?" If the student chose the answer out of four alternatives, "Spending a long time in reading a mathematics problem before solving it because I am not sure that the way I use to solve the problem was right", then the agent responded "Well, you may not feel confident about your work while solving the problem. Everyone's life is filled with uncertainty. However, with time and practice, you will find that worry will go away." If a student chose the answer "I always feel confident", the agent told the student to keep up with good work.

\section{Independent Variables}

There were three independent variables: anxiety reducing messages, learner gender, and the learner's prior anxiety. Anxiety messages had two levels of Presence vs. Absence. In the message presence condition, the agent presented both instructional guidance and anxiety messages. The agent's anxiety messages were presented in between sections (four to five sections per lesson) when the student progressed to a new concept. In the absence condition, the 
agent presented only instructional guidance. These two conditions were identical in all other aspects (e.g., information, problem solving practice, and feedback) and differed only in the anxiety messages. Learners' interaction logs showed no statistical difference in instructional time between the two conditions.

Learner gender was identified by self-report. At login, students were asked to indicate their gender (two levels, Male vs. Female). Also, prior to the study, student mathematics anxiety was measured. The scores were categorized into four levels: Low $(M=17.38, S D=1.28)$, Medium-low $(M=23.91, S D=2.38)$, Medium-high $(M=32.83, S D=3.02)$, High $(M=48.56$, $S D=10.46)$. According to the distribution of their mathematics anxiety scores $(M d n=29, I Q R=$ 18), the scores that were in the lowest quartile were categorized as low $(n=34)$; the scores that were in the highest quartile were categorized as high $(n=34)$; and the scores that were in the second and third quartiles were categorized as medium-low and medium-high, respectively $(n=$ 35 , for both groups).

\section{Measures}

\section{Mathematics anxiety}

The 16-item Learning Mathematics Anxiety scale, a subscale of Revised Mathematics Anxiety Rating Scale (RMARS), was used to measure learner anxiety in mathematics learning before and after intervention (Plake \& Parker, 1982). RMARS is targeted secondary school students and has been broadly used by researchers (Akin \& Kurbanoglu, 2011; Ashcraft, 2002; Devine et al., 2012; Leppavirta, 2011) and cited by more than one hundred studies since its development. The items used a five-point Likert scale ranging from 1 "not at all" to 5 "very much." Students were asked to indicate how anxious they were with activities, such as 1) Looking through the pages on a mathematics text, 2) Reading a formula, 3) Working on an 
abstract mathematical problem, and so forth. Inter-item reliability evaluated by Coefficient $\alpha$ was .91 in the pretest and .94 in the posttest.

\section{Mathematics learning}

Students' mathematics learning was measured with a pretest and an immediate posttest in each lesson. To ensure content validity of the test items, the items were developed concurrently when the lesson content was developed, so that the assessments matched what was expected to learn. Also, face validity was established through careful examination of the items by the researchers and collaborating mathematics teachers. Students were asked to solve a set of shortanswer problems in a pretest (6 questions in Lessons 1 through 3, 10 questions in Lesson 4); at the end of the lesson, they were asked to solve another set of equivalent questions that tested their understanding of matching concepts but were only different superficially. For example, in the lesson on Distributing Like Terms, students were asked to solve distributing $3(2 a+3 b)$ in the pretest and distributing $2(4 x+5 y)$ in the posttest. The Pearson correlation between pretest and posttest was $r=.79$, which indicated a high correlation. Students were allowed to use a scratch paper and a pencil. The items were scored for correctness, and no partial credit was awarded. The pre and posttests were implemented without agent presence.

\section{Procedures}

With the assistance of the algebra teachers, the research team implemented the four lessons using laptops in classrooms over a one-week period, one lesson per day with no lesson on Wednesday. On Day 1, the students were given a brief introduction to the lesson and the interfaces. Then, they were asked to put on the headset. They accessed the lesson web site and typed in their demographic information to $\log$ in. At login, they were randomly assigned to either the control group or the experimental condition. They took a mathematics anxiety pretest and a 
mathematics understanding pretest; following that, they continued to work on Lesson 1 guided by the tutor Chris (taking approximately 35-45 minutes with individual variations). Then they took a mathematics learning posttest. On Days 2-3, students started with a pretest covering the lesson topic, continued to work on the lesson, and took a posttest. On Day 4, they took a pretest, studied the lesson, and took a mathematics learning posttest and a mathematics anxiety posttest.

\section{Design and Analysis}

The research questions were answered using a pre and posttest experimental design. Our interest was to see if there would be any changes in mathematics anxiety and/or in learning gains from pretest to posttest following the intervention. A two-way repeated measures ANOVA was conducted for research questions 1-1 (on anxiety) and 1-2 (on learning), including two factors of treatment (message presence vs. absence) and gender (boys vs. girls). For research question 2-1 on anxiety, a two-way ANOVA was conducted to examine the effect of treatment and learner prior anxiety levels (high vs. mid vs. low). For research question 2-2 on learning, a two-way repeated measures ANOVA was conducted, including two factors of treatment and prior anxiety levels. Research questions 3-1 (on anxiety) and 3-2 (on learning) dealt only with high-anxiety students; a two-way repeated measures ANOVA was conducted including two factors of treatment and gender. The significance level was set at $\alpha<.05$ for all analyses.

Results

\section{Question 1: The Effect of Agent Anxiety Messages and Learner Gender}

\section{1-1) Do the anxiety messages and learner gender affect learners' mathematics anxiety?}

A two-way repeated measures ANOVA was conducted to test the effect of anxiety messages and learner gender on the anxiety of all participants, with anxiety messages and gender as between-subjects factors and time as a within-subjects factor. The repeated ANOVA revealed 
that there was a significant main effect of time on mathematics anxiety, $F(1,125)=4.205, M S E$ $=31.65, p=.042$. Students' anxiety decreased significantly from pretest to posttest after working at the weeklong agent-based lessons. The effect size of this difference, however, was small, as calculated by Cohen's $d, d=0.140$. There was no significant main effect of agent anxiety messages on anxiety, $F(1,125)=1.504, M S E=31.65, p=.222, \eta^{2}=.001$. There was no significant main effect of learner gender, $F(1,125)=0.120, M S E=31.65, p=.730, \eta^{2}=.000$. There was no significant interaction between treatment and learner gender, $F(1,125)=0.322$, $M S E=31.65, p=.572, \eta^{2}=.000$.

\section{1-2) Do the anxiety messages and learner gender affect learners' mathematics learning?}

A two-way repeated measures ANOVA was conducted to test the effect of anxiety messages and learner gender on the learning of all participants. The repeated ANOVA revealed that there was a significant main effect of time on mathematics learning of all participants, $F(1$, $134)=136.091, M S E=6.338, p<.001$. Students' learning increased significantly from pretest to posttest after working at the agent-based lessons. The effect size of this difference was medium, as calculated by Cohen's $d, d=.623$. There was no significant main effect of anxiety messages on learning, $F(1,134)=.180, M S E=6.338, p=.673, \eta^{2}=.000$. There was no significant main effect of gender, $F(1,134)=0.013, M S E=6.338, p=.910, \eta^{2}=.000$. There was no significant interaction effect of treatment and learner gender although the value was close to statistically significant, $F(1,134)=3.114, M S E=6.338, p=.080, \eta^{2}=.002$. Table 1 presents the means $(M)$ and standard deviations $(S D)$ of mathematics anxiety and mathematics learning scores by time, anxiety messages (treatment), and learner gender.

Question 2: The Effects of Agent Anxiety Messages and Learner Prior Anxiety 2-1) Do the anxiety messages and learners' prior anxiety levels affect their mathematics anxiety? 
A two-way ANOVA was conducted to test the effect of anxiety messages and learner prior anxiety levels on post-test mathematics anxiety of all participants. The two-way ANOVA revealed that there was a significant interaction effect between anxiety messages and prior anxiety levels, $F(3,120)=5.717, M S E=75.309, p=.001, \eta^{2}=.050$. However, this data set failed the homogeneity of variances assumption, so the result should be cautiously interpreted. Figure 3 presents this interaction trend graphically. By a visual inspection, high-anxiety students tended to demonstrate lower anxiety in the presence of the agent's anxiety messages than in the absence of the messages whereas the mid-range anxiety groups tended to demonstrate the opposite trend. We ran a Tukey's post-hoc analysis. The results revealed that the high-anxiety group neared statistical significance $(p<.07)$ whereas the other groups did not.

2-2) Do the anxiety messages and learners' prior anxiety levels affect their mathematics learning?

A two-way repeated ANOVA was conducted to test the effects of anxiety messages and learner prior anxiety levels on learning of all students. The results revealed that there was no significant main effect of prior anxiety on learning, $F(3,128)=0.868, M S E=6.427, p=.459, \eta^{2}$ $=.002$. There was also no significant interaction effect of treatment and prior anxiety, $F(3,128)$ $=.197, M S E=6.427, p=.899, \eta^{2}=.000$. Table 2 presents the means $(M)$ and standard deviations $(S D)$ of mathematics anxiety and mathematics learning scores by time, anxiety messages (treatment), and learner anxiety levels.

Question 3: The Effect of Anxiety Messages and Learner Gender for High-Anxiety Learners 3-1) Do the messages and learner gender affect highly anxious learners' mathematics anxiety? A two-way repeated ANOVA was conducted to test the effect of anxiety messages and learner gender only for highly anxious students. The results revealed that there was a significant main effect of time on anxiety, $F(1,25)=6.344, M S E=33.245, p=.019, \eta^{2}=.031$. Consistent 
with observations in Question 1 and Question 2, anxiety of the highly anxious group decreased from pretest to posttest after the weeklong agent-guided lessons. The effect size was small, as measured by Cohen's $d, d=.317$. There was neither significant main effect of anxiety messages, $F(1,25)=0.064, M S E=33.245, p=.802, \eta^{2}=.000$, nor learner gender on the anxiety of highanxiety students, $F(1,25)=1.320, M S E=33.245, p=.262, \eta^{2}=.007$. There was no significant interaction effect of anxiety messages and learner gender, $F(1,25)=0.958, M S E=33.245, p$ $=.337, \eta^{2}=.005$.

3-2) Do the messages and learner gender affect highly anxious learners ' mathematics learning?

Lastly, a two-way repeated measures ANOVA was conducted to test the effect of anxiety messages and student gender for high-anxiety students' learning. There was a significant main effect of time on learning, $F(1,28)=15.373, M S E=7.398, p<.001$. Learning of the highly anxious group increased significantly from pretest to posttest. The effect size was medium, as calculated using Cohen's $d, d=.489$. There was no significant main effect of anxiety messages on learning, $F(1,28)=.053, M S E=7.398, p=.473, \eta^{2}=.002$. There was also no significant main effect of gender on learning, $F(1,28)=.360, M S E=7.398, p=.553, \eta^{2}=.002$. There was no significant interaction effect of anxiety messages and gender on learning, $F(1,28)=.031$, $M S E=7.398, p=.860, \eta^{2}=.000$. Table 3 presents the means $(M)$ and standard deviations $(S D)$ of mathematics anxiety and mathematics learning scores by time, anxiety messages (treatment), and gender for high-anxiety students.

\section{Discussion}

Embodied agents can provide social and affective affordances in a variety of situations, and there is a growing body of research that attests to agents' effectiveness in developing students' positive affect and motivation to participate in learning tasks. (Baylor \& Kim, 2005; 
Kim, 2013; Kim \& Lim, 2013; Kim \& Wei, 2011; Moreno \& Mayer, 2000). Also, there is a consensus that mathematics anxiety is detrimental to learners' interest and ability in mathematics. Given the rarity of anxiety management in mathematics classrooms, the current study explored whether an embodied agent might be designed to help manage mathematics-related anxiety while guiding students' learning of mathematical concepts. The authors developed embodied-agentbased lessons equipped with anxiety-reducing messages adopted from popular clinical therapies as well as instructional guidance. The initial classroom experiments described in this paper did not result in strong evidence of the effectiveness of the agent's anxiety messages for all students. Rather, this trial revealed the efficacy of the agent-guided lessons that incorporated the solid instructional strategies. It seems that this finding underpins our initial effort to use embodied agent technology as a supplemental tool to manage learner anxiety in mathematics classrooms, therefore inviting continued efforts to refine the design of agent-based anxiety management.

\section{Agent Anxiety Treatment}

The statistical results did not confirm the benefit of the agent's anxiety reduction messages for all participants. Overall, the students decreased their anxiety from pretest to postest sigficantly after the weeklong agent-guided lessons, regardless of the presence or absence of the messages. The authors conjectured that the anxiety-management strategies were actually already embedded in the agents' instructional guidance. That is, following the literature on instructional strategies, the agent presented the concepts and problems from simple to complex step-by-step in a highly organized manner and also provided immediate, explanatory feedback that was tailored to typical learner errors. Also, learning from the agent who guided the participants personally and patiently through the lesson might have created a favorable, instructional context. Therefore, additional anxiety messages might only be necessary for students with high anxiety. This 
phenomenon was somewhat analogous to a previous study reporting that only the students with prior negative attitudes improved their attitudes toward mathematics learning significantly after working in agent-based learning (Kim, 2009). The current study showed that high-anxiety students benefited most from the agent's anxiety messages.

On the other hand, although the results were somewhat mixed for anxiety reduction, learning was constant regardless of the presence or absence of additional anxiety-reduction messages. All participants increased in their learning significantly after the agent-based lessons, and even the high-anxiety group's learning did not seem to be affected by the agents' messages or lack thereof. Similarly, some previous studies showed that agent effectiveness on learner affect was not always conductive to cognitive gains (Baylor \& Kim, 2005; Kim \& Wei, 2011). Perhaps this is because the consequence of any changes in psychological constructs might take longer to be realized, as represented in Fisher et al.'s study, which found changes in learner affect predicted performance improvement months later (Fisher, Dobbs-Oates, Doctoroff, \& Arnold, 2012). Likewise, the students who participated in this study, and particularly those with high anxiety, might show more significant emotional and cognitive gains related to their experience with embodied agents after a longer period of time ..

\section{Learner Gender}

Based on previous research presenting conflicting arguments on gender difference in mathematics anxiety, this study examined whether or not the agent's anxiety-reduction messages would influence girls and boys to a different degree. Our results did not reveal gender difference. Both boys' and girls' anxiety and learning were improved in a similar fashion after the agentbased lessons. This finding is in line with a recent study (Goetz et al., 2013) reporting that girls do not experience higher anxiety than boys do. 
Also, it is noteworthy that the previous research presenting gender difference has examined student anxiety in classroom instruction (Geist, 2010; Osborne, 2001). Often, researchers have attributed gender difference in motivation toward STEM learning to the unfavorable classroom atmosphere for girls (Cheryan, Plaut, Davies, \& Steele, 2009; Gallagher \& Kaufman, 2005; Sandler, Silverbert, \& Hall, 1996). However, such gender difference has not been observed in agent-based learning, or, at least, is less prominent. Rather, many agent studies have showed girls react more positively to an embodied agent than boys do (Arroyo, Burleson, Tai, Muldner, \& Woolf, 2013; Baylor \& Kim, 2004; Kim \& Lim, 2013). In this study, the agent 's individualized instruction might have induced favorable reactions from both genders, just as joyful facial expressions could elicit more positive affective responses (such as higher interest) regardless of learner gender (Ravaja \& Kätsyri, 2014). Hopefully, further research can demonstrate more clearly whether or not an embodied agent is able to supply an equitable context in learning mathematics.

\section{Implications and Recommendations}

Overall, the results of the study were in line with a trend prevalent in agent literature, confirming the affective benefit of humanlike agents and the need for a marriage between agent social presence and solid curricular pedagogy. Different from many agent studies examining ways to improve positive affect (e.g., interest, engagement, and self-efficacy), this study this study highlights the effect that agent-based learning can have on negative affect (e.g. anxiety).

The study had some limitations. The agent's anxiety messages were not truly personalized to each learner's needs: anxiety questions were asked with limited answer choices and agent responses pre-scripted by the choices. Also, as aforementioned, the weeklong intervention may have been too short to elicit all possible affective changes and consequential 
cognitive changes. Even when human therapists treat anxiety one on one, change is usually the result of a dedicated, long-term commitment. Additionally, there is no doubt that developing artificial beings that help reduce anxiety will be costly, demanding long-term resources. This study therefore could be viewed appropriately as a preliminary effort to try embodied agent technology to help anxious students in classrooms. Now that the study presented the initial evidence for agent effectiveness for high anxiety students, subsequent research might continue to refine agent-based anxiety treatment. First, agent treatment should become more adaptive to individuals' needs, taking advantage of advanced intelligent technology. Second, agent treatment might be deployed, at a minimum, for a few weeks or throughout an entire semester to genuinely understand the effectiveness of the treatment. Third, agent treatment might be tried out with a bigger subject pool, increasing the statistical power. On the other hand, in-depth qualitative data (e.g., interviews, observations, and survey responses) should complement the quantitative data to provide clearer information about the agent effectiveness on mathematical anxiety and learning.

To conclude, with the advance of technology, embodied agents might be able to provide personalized help for all learners. Many of today's adolescents have grown up with technologies as an integral part of their everyday life and are often called digital natives (Jones, Ramanau, Cross, \& Healing, 2010; Kennedy, Judd, \& Dalgarno, 2010; Prensky, 2001). In their use of the technologies, interacting with animated digital characters (e.g., avatars and agents) is becoming more ordinary (Turkle, 2011). Embodied agent technology could be effectively used as a supplemental tool for the young learners who grapple with everyday learning challenges and also alleviate pressure on overloaded teachers in classrooms.

\section{References}

Adams, C. (2001). Overcoming math anxiety. Mathematical Intelligencer, 23(1), 49-50. 
Akin, A., \& Kurbanoglu, I. N. (2011). The relationships between mathe anxiety, math attitudes, and self-efficacy: A structural equation model. Studia Psychologica, 53(3), 263-273.

Aksu, M., \& Saygi, M. (1988). The effects of feedback treatment on math-anxiety levels of sixth grade Turkish students. School Science and Mathematics, 88(5), 390-396.

Arroyo, I., Burleson, W., Tai, M., Muldner, K., \& Woolf, B. P. (2013). Gender differences in the use and benefit of advanced learning technologies for mathematics. Journal of Educational Psychology, 105(4), 957-969.

Ashcraft, M. H. (2002). Math anxiety: personal, educational, and cognitive consequences. Current Directions in Psychological Science, 11(5), 181-185.

Atkinson, R. K. (2002). Optimizing learning from examples using animated pedagogical agents. Journal of Educational Psychology, 94(2), 416-427. Retrieved from $<$ Go to ISI $>: / / 000176058300016$

Baker, D. (2002). Good intentions: An experiment in middle school single-sex science and mathematics classrooms with high minority enrollment. Journal of Women and Minorities in Science and Engineering, 8(1).

Baylor, A. L. (2011). The design of motivational agents and avatars. Educational Technology Research and Development, 59, 291-300.

Baylor, A. L., \& Kim, Y. (2004). Pedagogical agent design: The impact of agent realism, gender, ethnicity, and instructional role. Paper presented at the Intelligent Tutoring Systems, Maceió, Alagoas, Brazil.

Baylor, A. L., \& Kim, Y. (2005). Simulating instructional roles through pedagogical agents. International Journal of Artificial Intelligence in Education, 15, 95-115. 
Brownlow, S., Jacobi, T., \& Rogers, M. (2000). Science anxiety as a function of gender and experience. Sex Roles, 42, 119-131.

Cates, G. L., \& Rhymer, K. N. (2003). Examining the relationship between mathmatics anxiety and mathematics performance: An instructional hierarchy perspective. Journal of Behavioral Education, 12(1), 34.

Corbalan, G., Kester, L., \& van Merriëboer, J. J. G. (2009). Dynamic task selection: Effects of feedback and learner control on efficiency and motivation. Learning and Instruction, $19(6), 455-465$.

Devine, A., Fawcett, K., \& Szucs, D. e. a. (2012). Gender differences in mathematics anxiety and the relation to mathematics performance while controlling for test anxiety. Behavioral and Brain Functions, 8. doi:10.1186/1744-9081-8-33

Dihoff, R. E., Brosvic, G. M., Epstein, M. L., \& Cook, M. J. (2004). Provision of feedback during preparation for academic testing: Learning is enhanced by immediate but not delayed feedback. Psychological Record, 54(2), 207-232.

Dugas, M. J., \& Robichaud, M. (2006). Cognitive-behavioral treatment for generalized anxiety disorder: From science to practice. New York: Brunner-Routledge.

Dunsworth, Q., \& Atkinson, R. K. (2007). Fostering multimedia learning of science: Exploring the role of an animated agent's image. Computers \& Education, 49, 677-690.

Fisher, P. H., Dobbs-Oates, J., Doctoroff, G. L., \& Arnold, D. H. (2012). Early math interest and the development of math skills. Journal of Educational Psychology, 104(3), 673-681. doi:10.1037/a0027756

Foss, D. H., \& Hadfield, O. D. (1993). A successful clinic for the reduction of mathematics anxiety among college students. College Student Journal, 27(2), 157-166. 
Frenzel, A. C., Pekrun, R., \& Goetz, T. (2007). Girls and mathematics - A "hopeless" issue? A control-value approach to gender differences in emotions towards mathematics. European Journal of Psychology of Education, 22(4), 497-514.

Geist, E. (2010). The anti-anxiety curriculum: Combating math anxiety in the classroom. Journal of Instructional Psychology, 37(1), 24-31.

Goetz, T., Bieg, M., Lüdtke, O., Pekrun, R., \& Hall, N. C. (2013). Do girls really experience more anxiety in mathematics. Psychological Science, 24(10), 2079-2087.

Graesser, A. C., D'Mello, S. K., Craig, S. D., Witherspoon, A., Sullins, J., McDaniel, B., \& Gholson, B. (2008). The relationship between affective states and dialog patterns during interactions with AutoTutor. Journal of Interactive Learning Research, 19(2), 293-312.

Gulz, A. (2004). Benefits of virtual characters in computer-based learning environments: Claims and evidences. International Journal of Artificial Intelligence in Education, 14, 313-334.

Haak, D. C., HilleRisLambers, J., Pitre, E., \& Freeman, S. (2011). Increased structure and active learning reduce the achievement gap in introductory biology. Science, 332, 1213-1216. doi:10.1126/science. 1204820

Hattie, J., \& Timperley, H. (2007). The power of feedback. Review of Educational Research, $77(1), 81-112$.

Heidig, S., \& Clarebout, G. (2011). Do pedagogical agents make a difference to student motivation and learning? Educational Research Review, 6(1), 27-54.

Hembree, R. (1990). The nature, effects, and relief of mathematics anxiety. Journal for Research in Mathematics Education, 21(1), 33-46. 
Johnson, W. L., Rickel, J. W., \& Lester, J. C. (2000). Animated pedagogical agents: face-to-face interaction in interactive learning environments. International Journal of Artificial Intelligence in Education, 11, 47-78.

Jones, C., Ramanau, R., Cross, S. J., \& Healing, G. (2010). Net generation or digital natives: Is there a distinct new generation entering university? Computers \& Education, 54(3), 722732.

Kennedy, G., Judd, T., \& Dalgarno, B. (2010). Beyond Natives and Immigrants: Exploring types of net generation students. Journal of Computer Assisted Learning, 26(5), 332-343.

Kim, Y. (2009). The role of learner attributes and affect determining the impact of agent presence. International Journal of Learning Technology, 4(3), 234-249.

Kim, Y. (2013). Digital peers to help children's text comprehension and perceptions. Journal of Educational Technology \& Society, 16(4), 59-70.

Kim, Y., \& Lim, J. (2013). Gendered socialization with an embodied agent: Creating a social and affable mathematics learning environment for middle-grade females. Journal of Educational Psychology, 105(4), 1164-1174.

Kim, Y., \& Wei, Q. (2011). The impact of user attributes and user choice in an agent-based environment. Computers \& Education, 56, 505-514.

Kirschner, P. A., Sweller, J., \& Clark, R. E. (2006). Why minimal guidance during instruction does not work: An analysis of the failure of constructivist, discovery, problem-based, experiential, and inquiry-based teaching. Educational Psychologist, 41(2), 75-86.

Krämer, N. C., \& Bente, G. (2010). Personalizing e-Learning. The social effects of pedagogical agents. Educational Psychology Review, 22(1), 71-87. 
Krämer, N. C., Kopp, S., Becker-Asano, C., \& Sommer, N. (2013). Smile and the world will smile with you — The effects of a virtual agent's smile on users' evaluation and behavior. International Journal of Human Computer Studies, 71, 335-349.

Leppavirta, J. (2011). The Impact of mathematics anxiety on the performance of students of electromagnetics. Journal of Engineering Education, 100(3), 424-443

Lester, J. C., Callaway, C. B., Gregoire, J., Stelling, G., Towns, S. G., \& Zettlemoyer, L. (2001). Animated pedagogical agents in knowledge-based learning environments. In K. D. Forbus \& P. J. Feltovich (Eds.), Smart machines in education (pp. 71-97). Cambridge, MA: The MIT Press.

Liebert, R., \& Morris, L. (1967). Cognitive and emotional components of test anxiety: A distinction and some initial data. Psychological Reports, 20, 975-978.

Lindberg, S. M., Hyde, J. S., Petersen, J. L., \& Linn, M. C. (2010). New trends in gender and mathematics performance: A meta-analysis. Psychological Bulletin, 136(6), 1123-1135.

Ma, X. (1999). A meta-analysis of the relationship between anxiety toward mathematics and achievement in mathematics. Journal for Research in Mathematics Education, 30(5), $520-540$.

Ma, X., \& Xu, J. (2004). The causal ordering of mathematics anxiety and mathematics achievement: a longitudinal panel analysis. Journal of Adolescence, 27(2), 165-179.

McKendree, J. (1990). Effective feedback content for tutoring complex skills. Human-Computer Interaction, 5(4), 381-413.

Moreno, R., \& Mayer, R. E. (2000). A coherence effect in multimedia learning: The case for minimizing irrelevant sounds in the design of multimedia instructional messages. Journal of Educational Psychology, 97, 117-125. 
Moreno, R., Mayer, R. E., Spires, H. A., \& Lester, J. C. (2001). The case for social agency in computer-based teaching: do students learn more deeply when they interact with animated pedagogical agents? Cognition and Instruction, 19(2), 177-213.

Morris, L. W., Davis, M. A., \& Hutchings, C. H. (1981). Cognitive and emotional components of anxiety: Literature review and a revised worry-emotionality scale. Journal of Educational Psychology, 73, 541-555.

Morsanyi, K., Busdraghi, C., \& Primi, C. (2014). Mathematical anxiety is linked to reduced cognitive reflection: a potential road from discomfort in the mathematics classroom to susceptibility to biases. Behavioral and Brain Functions, 10(31), http://www.behavioralandbrainfunctions.com/content/10/11/31.

Moundridou, M., \& Virvou, M. (2002). Evaluating the Persona Effect of an Interface Agent in a Tutoring System. Journal of Computer Assisted Learning, 18, 253-261.

Mulken, S. V., Andre, E., \& Muller, J. (1998). The Persona Effect: How Substantial Is It? Paper presented at the International Conference of Computer Human Interaction (CHI), Berlin, Germany.

Nass, C., Roles, E., \& Wang, Q. (2005). "User as assessor" approach to embodied conversational agents: The case of apparent attention in ECAs. In Z. Ruttkay \& C. Pelachaud (Eds.), From brows to trust: Evaluating embodied conversational agents: Springer.

Norwood, K. S. (1994). The effect of instructional' approach on mathematics anxiety and achievement. School Science and Mathematics, 94(5), 248-254.

Osborne, J. W. (2001). Testing stereotype threat: Does anxiety explain race and sex differences in achievement? Contemporary Educational Psychology, 26, 291-310. 
Preis, C., \& Biggs, B. T. (2001). Can instructors help learners overcome math anxiety? American Technical Education Association Journal, 28(4), 6-10.

Prensky, M. (2001). Digital natives digital immigrants. On the Horizon, 9.

Ravaja, N., \& Kätsyri, J. (2014). Suboptimal facial expression primes in textual media messages: Evidence for the affective congruency effect. Computers in Human Behavior, 40, 64-77.

Reyes, M. R., Brackett, M. A., Rivers, S. E., White, M., \& Salovey, P. (2012). Classroom emotional climate, student engagement, and academic achievement. Journal of Educational Psychology, 104(3), 700-712. doi:10.1037/a0027268

Robertson, J., Cross, B., Macleod, H., \& Wiemer-Hastings, P. (2004). Children's interactions with animated agents in an Intelligent tutoring system. International Journal of Artificial Intelligence in Education, 14, 335-357.

Ryokai, K., Vaucelle, C., \& Cassell, J. (2003). Virtual peers as partners in storytelling and literacy learning. Journal of Computer Assisted Learning, 19(2), 195-208.

Steele, D. E., \& Arth, A. A. (1998). Lowering anxiety in the math curriculum. Education Digests, 63(7), 18-23.

Turkle, S. (2011). Alone together: Why we expect more from technology and less from each other. New York: Basic Books.

Wadlington, E., Austin, S., \& Bitner, J. (1992). The treatment of math anxiety and negative math self-concept in college students. College Student Journal, 26(1), 61-65.

Wang, N., Johnson, W. L., Mayer, R. E., Rizzo, P., Shaw, E., \& Collins, H. (2008). The politeness effect: Pedagogical agents and learning outcomes. International Journal of Human-Computer Studies, 66, 98-112. 


\section{Table 1}

M's and SD's of Anxiety and Learning by Time, Treatment, and Gender for All Participants

\begin{tabular}{ccccccccccc}
\hline & & \multicolumn{4}{c}{ Anxiety $^{1}$} & \multicolumn{4}{c}{ Learning } \\
\cline { 3 - 11 } & & \multicolumn{2}{c}{ Pretest } & \multicolumn{2}{c}{ Posttest } & \multicolumn{2}{c}{ Pretest } & \multicolumn{2}{c}{ Posttest } \\
\hline Gender & Anxiety Messages & $M$ & $S D$ & $M$ & $S D$ & $M$ & $S D$ & $M$ & $S D$ \\
\hline \multirow{2}{*}{$\begin{array}{c}\text { Male } \\
(n=59)\end{array}$} & Presence $(n=31)$ & 29.58 & 12.16 & 28.84 & 13.32 & 16.97 & 6.20 & 21.18 & 6.52 \\
\cline { 2 - 11 } & Absence $(n=28)$ & 28.61 & 11.41 & 26.93 & 13.01 & 17.54 & 5.95 & 20.42 & 6.21 \\
\hline $\begin{array}{c}\text { Female } \\
(n=70)\end{array}$ & Presence $(n=28)$ & 28.54 & 8.31 & 28.11 & 10.90 & 16.50 & 5.12 & 19.70 & 5.61 \\
\cline { 2 - 10 } & Absence $(n=42)$ & 30.00 & 12.01 & 27.02 & 11.02 & 15.82 & 5.53 & 19.84 & 5.70 \\
\hline \multirow{2}{*}{} & Total $^{*}$ & 29.28 & 11.10 & 27.67 & 11.91 & 16.63 & 5.68 & 20.26 & 5.96 \\
\hline
\end{tabular}

$p<.01$

${ }^{1}$ Anxiety score ranges: $16-80$; Learning score ranges: $0-28$. 
Table 2

M's and SD's of Mathematics Anxiety by Time, Treatment, and Prior Anxiety Levels

\begin{tabular}{|c|c|c|c|c|c|c|c|c|c|}
\hline \multirow[b]{3}{*}{$\begin{array}{c}\text { Prior } \\
\text { Anxiety }\end{array}$} & \multirow[b]{3}{*}{$\begin{array}{c}\text { Anxiety } \\
\text { Messages }\end{array}$} & \multicolumn{4}{|c|}{ Anxiety $^{1}$} & \multicolumn{4}{|c|}{ Learning } \\
\hline & & \multicolumn{2}{|c|}{ Pretest } & \multicolumn{2}{|c|}{ Posttest } & \multicolumn{2}{|c|}{ Pretest } & \multicolumn{2}{|c|}{ Posttest } \\
\hline & & $M$ & $S D$ & $M$ & $S D$ & $M$ & $S D$ & $M$ & $S D$ \\
\hline \multirow{3}{*}{$\begin{array}{c}\text { High } \\
(n=32)\end{array}$} & Presence $(n=13)$ & 46.31 & 5.59 & 40.38 & 10.99 & 15.27 & 5.64 & 18.40 & 6.20 \\
\hline & Absence $(n=19)$ & 49.95 & 12.54 & 49.47 & 15.66 & 16.21 & 6.20 & 18.63 & 6.34 \\
\hline & Total & 48.47 & 10.33 & 45.78 & 14.34 & 15.79 & 5.88 & 18.53 & 6.18 \\
\hline \multirow{3}{*}{$\begin{array}{l}\text { Medium- } \\
\text { high } \\
(n=33)\end{array}$} & Presence $(n=16)$ & 32.69 & 3.09 & 34.56 & 11.43 & 17.88 & 5.90 & 22.06 & 5.79 \\
\hline & Absence $(n=17)$ & 32.71 & 3.06 & 28.84 & 4.98 & 17.47 & 4.98 & 21.21 & 6.06 \\
\hline & Total & 32.70 & 3.03 & 30.79 & 9.35 & 17.66 & 5.34 & 21.60 & 5.87 \\
\hline \multirow{3}{*}{$\begin{array}{l}\text { Medium-low } \\
\quad(n=33)\end{array}$} & Presence $(n=15)$ & 24.67 & 2.50 & 24.80 & 7.31 & 17.44 & 5.67 & 21.38 & 6.52 \\
\hline & Absence $(n=18)$ & 23.39 & 2.19 & 19.33 & 2.93 & 15.12 & 6.54 & 19.00 & 6.51 \\
\hline & Total & 23.97 & 2.39 & 21.82 & 5.97 & 16.17 & 6.18 & 20.09 & 6.53 \\
\hline \multirow{3}{*}{$\begin{array}{c}\text { Low } \\
(n=30)\end{array}$} & Presence $(n=14)$ & 17.36 & 1.22 & 16.50 & 0.94 & 16.31 & 5.68 & 19.94 & 5.81 \\
\hline & Absence $(n=16)$ & 17.38 & 1.41 & 16.81 & 1.56 & 18.50 & 4.12 & 22.69 & 2.55 \\
\hline & Total & 17.37 & 1.30 & 16.67 & 1.30 & 17.41 & 5.00 & 21.31 & 4.63 \\
\hline
\end{tabular}

${ }^{1}$ Anxiety score ranges: $16-80$; Learning score ranges: 0 - 28 . 
Table 3

M's and SD's of Anxiety by Time, Treatment, and Gender for High-Anxiety Students

\begin{tabular}{|c|c|c|c|c|c|c|c|c|c|}
\hline \multirow[b]{3}{*}{ Gender } & \multirow[b]{3}{*}{ Anxiety Messages } & \multicolumn{4}{|c|}{ Anxiety $^{1}$} & \multicolumn{4}{|c|}{ Learning } \\
\hline & & \multicolumn{2}{|c|}{ Pretest } & \multicolumn{2}{|c|}{ Posttest } & \multicolumn{2}{|c|}{ Pretest } & \multicolumn{2}{|c|}{ Posttest } \\
\hline & & $M$ & $S D$ & $M$ & $S D$ & $M$ & $S D$ & $M$ & $S D$ \\
\hline \multirow{2}{*}{$\begin{array}{c}\text { Male } \\
(n=15)\end{array}$} & Presence $(n=7)$ & 46.00 & 4.28 & 45.00 & 6.71 & 15.33 & 6.78 & 18.33 & 7.30 \\
\hline & Absence $(n=8)$ & 53.75 & 16.25 & 50.50 & 19.37 & 20.43 & 4.47 & 22.14 & 4.18 \\
\hline \multirow{3}{*}{$\begin{array}{l}\text { Female } \\
(n=14)\end{array}$} & Presence $(n=5)$ & 46.00 & 7.91 & 38.40 & 9.76 & 13.80 & 2.28 & 17.40 & 4.34 \\
\hline & Absence $(n=9)$ & 47.33 & 9.50 & 43.56 & 6.37 & 14.82 & 4.73 & 17.64 & 5.78 \\
\hline & Total & $48.55^{*}$ & $10.75^{*}$ & $44.93^{*}$ & $12.07^{*}$ & $16.03^{* * *}$ & $5.43^{* \star *}$ & $18.78^{* * *}$ & $5.81^{* *}$ \\
\hline
\end{tabular}

$p<.05 ; \quad p<.001$

${ }^{1}$ Anxiety score ranges: $16-80$; Learning score ranges: $0-28$. 


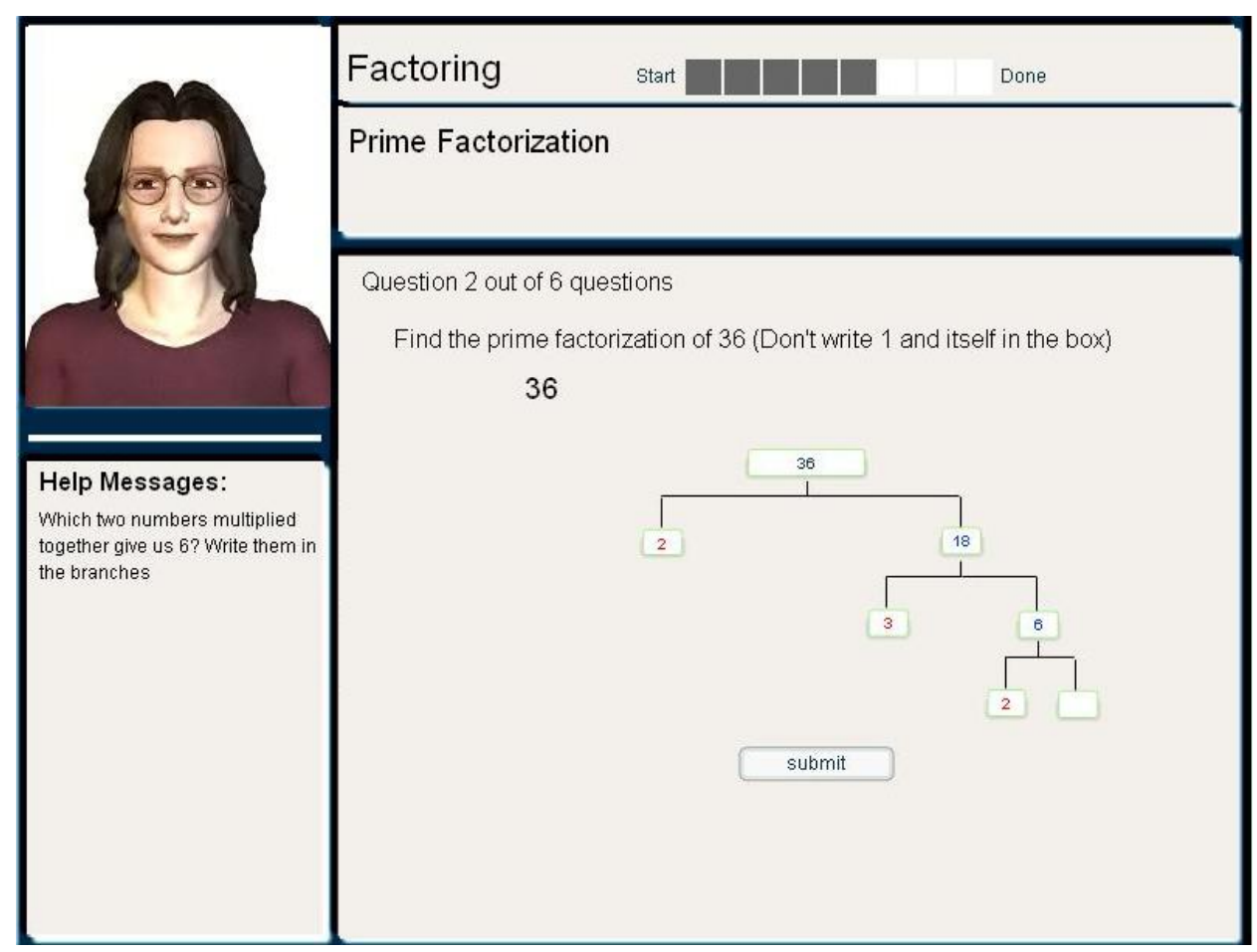

Figure 1. A screen sample of agent-based lessons. 


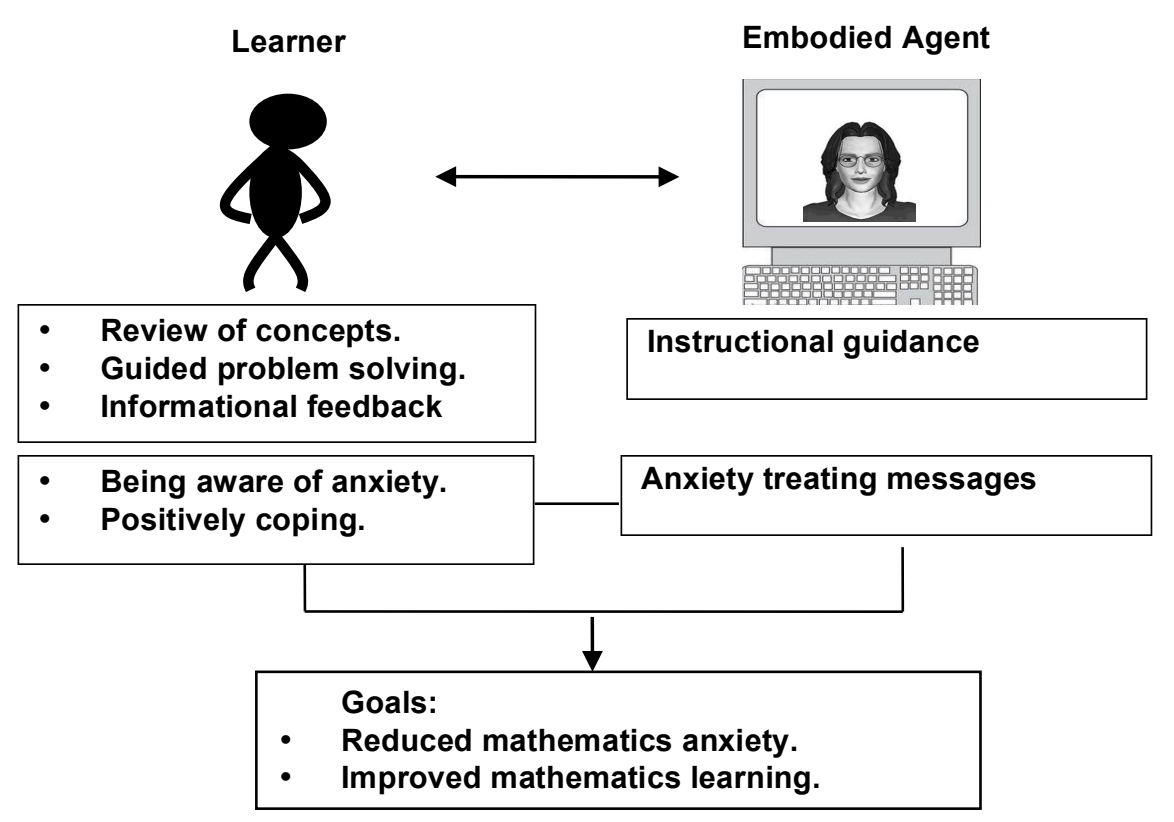

Figure 2. Agent/learner interaction diagram. 


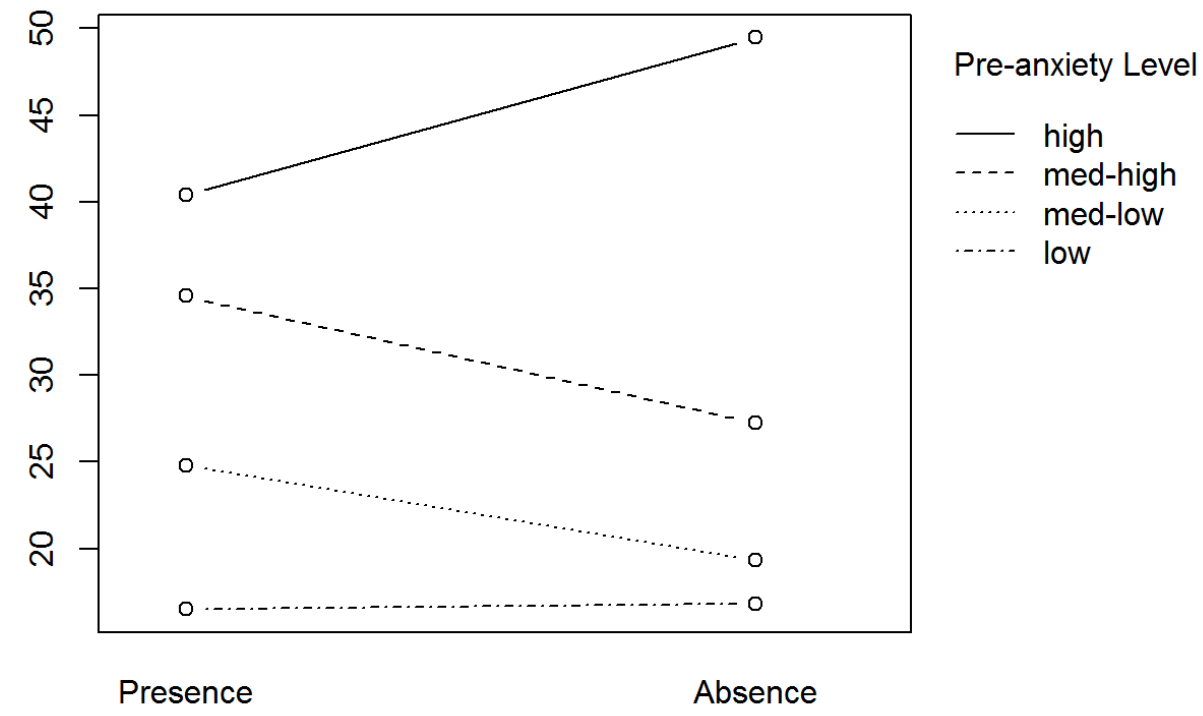

Anxiety Messages

Figure 3. An interaction trend of agent messages and learner prior anxiety. 